\title{
POSITIONS OF THE RANKS OF FACTORS IN CERTAIN FINITE LONG LENGTH WORDS
}

\author{
ELAHE ZOHOORIAN AZAD
}

We consider the set of finite random words $\mathcal{A}^{\star}$, with independent letters drawn from a finite or infinite totally ordered alphabet according to a general probability distribution. On a specific subset of $\mathcal{A}^{\star}$, considering certain factorization of the words which are labelled with the ranks, base on the lexicographical order, we prove that the normalized position of the ranks of factors, are uniform, when the length of the word goes to infinity.

\section{INTRODUCTION}

We consider a general probability distribution $\left(p_{i}\right)_{i \geq 1}\left(p_{i}>0\right)$ on a set $\mathcal{A}=$ $\left\{a_{1}<a_{2}<\ldots\right\}$ of letters, and we assume, without loss of generality, that $0<p_{1}<$ 1. On the corresponding set of words, $\mathcal{A}^{\star}=\bigcup_{n>0} \mathcal{A}_{n}\left(\mathcal{A}_{n}\right.$ the set of words with length $n$ ), considering the word $w \in \mathcal{A}_{n}$ that can be expressed as $w=a_{\ell_{1}} a_{\ell_{2}} \ldots a_{\ell_{n}}$ $\left(\ell_{i}\right.$ 's are the positive integer numbers), we define the weight $p(w)$ as

$$
p(w)=p_{\ell_{1}} p_{\ell_{2}} \ldots p_{\ell_{n}} .
$$

With the weight $p($.$) , comes a probability measure on \mathcal{A}_{n}, \mathbb{P}_{n}(\{w\})=p(w)$, and thus a probability measure on any subset of $\mathcal{A}_{n}$. Letting $\mathfrak{F}_{n}$ denotes the $\sigma$-algebra generated by $\mathcal{A}_{n}$, the triple of $\left(\mathcal{A}_{n}, \mathfrak{F}_{n}, \mathbb{P}_{n}\right)$ is the corresponding probability space.

We recall here some general definitions from [3] (readers can see also [8, 4, 5]). A word $v$ is a factor of a word $w$ if there exists two other words $s$ and $t$, possibly empty, such that $w=s v t$. If $s$ is empty $v$ is a prefix (or a right factor) of $w$ and if $t$ is empty $v$ is a suffix (or a left factor) of $w$.

A lexicographic order on the set of words $\mathcal{A}^{\star}$ is given by a total order on the alphabet $\mathcal{A}=\left\{a_{1}, a_{2}, \ldots\right\}$ extended to the words in the following way: A word $u$, is said to be smaller than a word $v$ if $u$ is a prefix of $v$ or $u=r a_{i} s$ and $v=r a_{j}$ t such that $i<j$ and $r, s$ and $t$ be some words, possibly empty.

For $w=w_{1} \ldots w_{n}$, a word in $\mathcal{A}_{n}(n>0)$, we define $\tau w=w_{2} \ldots w_{n} w_{1}$. Then $\langle\tau\rangle=\left\{I d, \tau, \ldots, \tau^{n-1}\right\}$ is the group of cyclic permutations of the letters of a word with length $n$. The orbit $\langle w\rangle$ of a word $w$ under $\langle\tau\rangle$ is called a necklace.

A word $w \in \mathcal{A}_{n}(n>0)$ is called primitive if its necklace $\langle w\rangle$ has exactly $n$ elements. In other words, a word $w \in \mathcal{A}_{n}$ is primitive if it is not a power of another word in $\mathcal{A}^{*}$ (remark that a word $w$ is a power of another word $u$, if $w$ can be written as $w=u u u \ldots u)$. Denote by $\mathcal{P}_{n}$ the set of primitive words in $\mathcal{A}_{n}$ and by $\mathcal{N}_{n}$ its complement.

In this article we work on a subset of $\mathcal{P}_{n}(n>0)$ containing the primitive words which begin with a run of the own smallest letter and end with a run of

1991 Mathematics Subject Classification. 68R15, 60B10, 68Q25. 
a letter different from the smallest. We denote this subset by $\mathcal{W}_{n}$. For example the word $a_{2} a_{3} a_{4} a_{2} a_{3}$ is a word in $\mathcal{W}_{5}$. The set of $\mathcal{W}_{n}$ contains the words with certain properties that can be interesting in some applications of combinatorial on words. The Lyndon words with length $n$ (the words which are strictly smaller than any their proper suffix), for example, are included in $\mathcal{W}_{n}$. We consider then the probability measure $\mathbb{W}_{n}$ on $\mathcal{W}_{n}$ (the conditioning probability in the probability space of $\left.\left(\mathcal{A}_{n}, \mathfrak{F}_{n}, \mathbb{P}_{n}\right)\right)$ :

$$
\mathbb{W}_{n}(\{w\})=\frac{\mathbb{P}_{n}(\{w\})}{\mathbb{P}_{n}\left(\mathcal{W}_{n}\right)} .
$$

Now, we divide the words of $\mathcal{W}_{n}$ to the factors that we call the blocks of word, in the following way:

Definition 1.1. Let $w$ be a word in $\mathcal{W}_{n}$ and $a_{w}$ the smallest letter of $w$ (remark that $w$ begins with $a_{w}$ ). The blocks of $w$ are the factors of $w$ that begin with a run of " $a_{w}$ " and end just before very next run of " $a_{w}$ ".

Thus, the blocks of a word are the factors in the form $a_{w}{ }^{k_{0}} a_{\ell_{1}}^{k_{1}} a_{\ell_{2}}^{k_{2}} \ldots a_{\ell_{m}}^{k_{m}}$ such that $a_{\ell_{i}} \neq a_{w}$ and $k_{0}>0$.

Now, concerning the lexicographical order, each block of a word $w$ in $\mathcal{W}_{n}$ can be ranked, according to the order of the word in the necklace of $\langle w\rangle$ which begins by the mentioned block. For example, in the word $w=a c a^{2} b a c d b a^{2} b a^{3} d^{2} b$, the blocks and their related ranks below them, are:

$$
\begin{array}{cccc}
a c, a^{2} b, a c d b, & a^{2} b, a^{3} d^{2} b,
\end{array}
$$

as there is the following order between the five words of the necklace $\langle w\rangle$, which begin by the blocks of $w$ :

$$
\begin{aligned}
a^{3} d^{2} b a c a^{2} b a c d b a^{2} b & \leq a^{2} b a^{3} d^{2} b a c a^{2} b a c d b \leq a^{2} b a c d b a^{2} b a^{3} d^{2} b a c \\
& \leq a c a^{2} b a c d b a^{2} b a^{3} d^{2} b \leq a c d b a^{2} b a^{3} d^{2} b a c a^{2} b .
\end{aligned}
$$

In this work, we are interested in the limiting distribution of the positions of ranks of a random word of $\mathcal{W}_{n}$, which seems to be uniform. It is trivial that if the uniform random permutation of the blocks, causes the uniform displacements of the ranks, the purpose would be entailed. But in general, it is not true because of the existence of the equal blocks. In fact, it is well possible that certain permutations of ranks are not produced. For example, for the word $w=a^{2} b, a^{2} b, a b, a b$ with the respective ranks $1,2,4,3$ of its blocks, the permutation $1,2,3,4$ of the ranks is not produced by any permutation of the blocks of $w$, no more any permutation of the cyclic permutation of $1,2,3,4$ as $4,1,2,3$. Moreover, certain permutations of the blocks of a word, like the permutation $a^{2} b, a b, a^{2} b, a b$ of the blocks of $w$, produce the non-primitive words for which the ranks of the blocks are not defined. Nevertheless, by definition of the ranks, it is trivial that the cyclic permutations of the blocks of a word causes the cyclic permutations of the related ranks. On the other hand, in any orbit of the cyclic permutation of the ranks, the ranks are uniformly distributed on all positions; the fact that is the key of the prove.

Marchand \& Zohoorian in [6, Section 6], for a random word of $\{a, b\}^{n}$ when $n$ is sufficiently large, demonstrate hardly that the uniform permutation of the blocks 
of the word, barring the block with rank 1, entails the uniform displacement of the rank 2 on all possible positions. However, our result may be applied also in the analyze of the height of the labelled binary trees emerged by the successive iterations of the standard factorization of the Lyndon words (an introduction on Lyndon trees is given by Marchand \& Zohoorian in [6, Section 1] or by Bassino et al. in [1]). In fact, the height of Lyndon trees has a direct relation with the positions of the ranks in the root word. The study on the structure of Lyndon trees is a work in progress. The main theorem that we will prove in this article is:

Theorem 1.1. The positions of ranks in a random word of $\mathcal{W}_{n}$, divided by $\frac{p_{1}\left(1-p_{1}\right) n}{2}$, converge in law, when $n$ goes to infinity, to

$$
\mu(d x)=\mathbf{1}_{[0,1]}(x) d x,
$$

where $d x$ denotes the Lebesgue measure on $\mathbb{R}$.

\section{NUMBER OF THE BLOCKS OF A WORD}

To begin, we concentrate on the number of the blocks of a random word in $\mathcal{W}_{n}$. It is evident that the number of the blocks of a word in $\mathcal{W}_{n}$ is equal to the number of the runs of its smallest letter. Now, we define the following function on $\mathcal{P}_{n}$ that carries the words of $\mathcal{P}_{n}$ to its subset $\mathcal{W}_{n}$ :

Definition 2.1. Let $\phi$ denotes the function on $\mathcal{P}_{n}$ that brings any word $w$ of $\mathcal{P}_{n}$ to itself if $w$ is a word in $\mathcal{W}_{n}$, and otherwise to the word of $\langle w\rangle$ which begins by the last run of the smallest letter of $w$ (see the following example).

\section{Example.}

- If $w=a b a^{2} c b^{2} a^{2}$, then $\phi(w)=a^{3} b a^{2} c b^{2}$.

- If $A=\left\{a b a^{2} c, a c b a b\right\} \subset \mathcal{W}_{5}$ then $\phi^{-1}(A)=\left\{a b a^{2} c, b a^{2} c a\right\} \cup\{a c b a b, c b a b a, b a b a c\}$.

We remark that $\phi$ is a surjective map on $\mathcal{P}_{n}$ to $\mathcal{W}_{n}$ and the inverse image of $w \in \mathcal{W}_{n}$ is a subset of $\mathcal{P}_{n}$ whose cardinality is equal to the length of the first block of $w$.

The next lemma allows to transfer results from random words of $\mathcal{A}_{n}$ to random words of $\mathcal{W}_{n}$. For this mean, we set

$$
\|p\|_{\alpha}=\left(\sum_{i} p_{i}^{\alpha}\right)^{1 / \alpha}
$$

for $\alpha \geq 1$.

Notation. If $a$ and $b$ are two functions from $\mathbb{N}$ into $\mathbb{R}, a(n)=\mathcal{O}(b(n)) \Leftrightarrow \exists c>$ $0, \forall n \in \mathbb{N},|a(n)| \leq c|b(n)|$.

Lemma 2.1. For $A \subset \mathcal{W}_{n}$, we have:

$$
\left|\mathbb{W}_{n}(A)-\mathbb{P}_{n}\left(\phi^{-1}(A)\right)\right|=\mathcal{O}\left(\|p\|_{2}^{n}\right) .
$$

Note that $\|p\|_{1}=1$, and that, under the assumption $\left\{0<p_{1}<1\right\},\|p\|_{\alpha}$ is strictly decreasing in $\alpha$. Other well known inequalities include $\|p\|_{2} \leq \sqrt{\max p_{i}}$. 
The lines of the proof of this lemma are exactly the same as in the proof of Lemma 2.1 of [2], but here we work on the set $\mathcal{W}_{n}$, instead of the set of Lyndon words, there. So we remove the proof, referring the reader to [2, Lemma 2.1].

Let now for any word $w$ in $\mathcal{A}_{n}$ we denote by $N_{n}^{a_{1}}(w)$ the number of runs of the letter $a_{1}$ in the word $w$. We have then the following lemma:

Lemma 2.2. (Number of runs of the letter $a_{1}$ ).

$$
\mathbb{W}_{n}\left(N_{n}^{a_{1}}<\frac{p_{1}\left(1-p_{1}\right)}{2} n\right)=\mathcal{O}\left(n^{-1}\right) .
$$

We remove the proof of this lemma, again inviting the reader to see [2, Lemma $2.3]$.

By Lemma 2.2, one sees that the number of the blocks of a word with length $n$, when $n$ is sufficiently large, is of the order $n$ with a high probability as a word with length $n$ has at most $\frac{n}{2}$ blocks (the case where the smallest letter repeats alternatively).

\section{Displacements of RANKS}

We consider, at first, some notations and definitions. As a consequence of Definition 1.1, any word $w \in \mathcal{W}_{n}$ can be decomposed uniquely as

$$
w=B_{1}(w), B_{2}(w), \ldots, B_{N_{n}(w)}(w),
$$

in which $B_{i}(w)$ 's stand for the blocks of $w$ and $N_{n}(w)$ denotes the number of the blocks. We denote the respective ranks of the blocks of $w$ by

$$
r(w)=r_{1}(w), r_{2}(w), \ldots, r_{N_{n}(w)}(w),
$$

called, briefly, the rank of $w$. Obviously, $r(w)$ is a permutation of $1,2, \ldots, N_{n}(w)$.

Definition 3.1. For any $w=B_{1}, B_{2}, \ldots, B_{N} \in \mathcal{W}_{n}$ in which $B_{i}$ 's are the blocks of $w$, we define $\beta w=B_{2}, \ldots, B_{N}, B_{1}$. Then $\langle\beta\rangle=\left\{I d, \beta, \ldots, \beta^{N-1}\right\}$ is the group of cyclic permutations of the blocks of a word in $\mathcal{W}_{n}$. We call the orbit $\langle w\rangle_{\beta}$ of the blocks of $w$ under $\langle\beta\rangle$, the block orbit of $w$.

Proposition 3.1. The ranks of the blocks of a word in $\mathcal{W}_{n}$ are invariant under the cyclic permutation of the blocks.

Proof. By definition of the ranks, it is evident that the ranks are permuted cyclically as the blocks are permuted.

The following corollary is an immediate result of the above proposition and the proof is left as it is evident.

Corollary 3.1. In any block orbit, the ranks are distributed uniformly on all positions. In other words, if the number of the words in a block orbit is $N$, the probability that the $i$-th rank, $i=1, \ldots, N$, be in the position $k, k=1, \ldots, N$, is equal to $\frac{1}{N}$.

Let $\mathfrak{S}_{n}$ denote the set of permutations of $\{1, \ldots, n\}$. For $w \in \mathcal{W}_{n}$, and $\sigma \in$ $\mathfrak{S}_{N_{n}(w)}$, we set

$$
\sigma \cdot w=B_{\sigma(1)}(w), \ldots, B_{\sigma\left(N_{n}(w)\right)}(w) .
$$

Conditioning then $\sigma . w \in \mathcal{W}_{n}$, the rank of $\sigma . w, r(\sigma . w)$, is also a permutation in $\mathfrak{S}_{N_{n}(w)}$. We set $C(w)=\left\{\sigma . w: \sigma \in \mathfrak{S}_{N_{n}(w)} \& \sigma . w \in \mathcal{W}_{n}\right\}$ and $\mathcal{C}_{n}=$ 
$\left\{C(w): w \in \mathcal{W}_{n}\right\}$. Let $\mathfrak{C}_{n}$ denote the $\sigma$-algebra generated by $\mathcal{C}_{n}$. In the following proposition we see that $\mathcal{W}_{n}$ is parted to $C(w) \mathrm{s}$ :

Proposition 3.2. $\mathcal{C}_{n}$ is a partition of $\mathcal{W}_{n}$.

Proof. Assume that $w \in \mathcal{W}_{n}$, and $w^{\prime} \in C(w)$ : then $w^{\prime} \in \mathcal{W}_{n}$ and $w^{\prime}$ has the same multiset of blocks as $w$ (it has the same blocks, with the same multiplicity). As a consequence, for $w, w^{\prime} \in \mathcal{W}_{n}$, either $C(w)=C\left(w^{\prime}\right)$ or $C(w) \cap C\left(w^{\prime}\right)=\emptyset$.

In the following proposition, which is the key proposition of this result, we see that the block orbits divide $C(w)$ :

Proposition 3.3. For any $w \in \mathcal{W}_{n}, C(w)$ is parted to the block orbits.

Proof. As $C(w)$ contains the primitive words produced by permutations of the blocks of $w$, it is sufficient to verify that all cyclic permutations of the blocks of a word in $\mathcal{W}_{n}$ produce the primitive words. Suppose now that a cyclic permutation of the blocks of a word $v=B_{1}, B_{2}, \ldots, B_{N} \in \mathcal{W}_{n}$, for example $\beta^{i} v=$ $B_{i+1}, B_{i+2}, \ldots, B_{N}, B_{1}, \ldots, B_{i}(1 \leq i \leq N)$, is a non-primitive word in the form $u^{r}$ for $u \in \mathcal{A}^{*}$ and $2 \leq r \leq n$. As $u$ is a prefix and a suffix of $\beta^{i} v$ and $\beta^{i} v$ is a word in $\mathcal{W}_{n}, u$ is a word which begins by its smallest letter and end with a letter different from the smallest letter. Therefore $u$ can be factorized in the blocks and so has exactly the same blocks as the first blocks of $\beta^{i} v$. Consequently, the blocks of $\beta^{i} v$ are periodically equals. That is, if $u$ has $j$ number of the blocks, they are equals to the first $j$, second $j, \ldots$ and last $j$ blocks of $\beta^{i} v$. But this equalities, in $\beta^{i} v$, entail that for any $k=1,2, \ldots, N-j, B_{k}=B_{k+j}$ and that all cyclic permutations of the blocks of $\beta^{i} v$, especially $v$, will be the words which can be part to $\frac{N}{j}$ equal factors. Therefore, any cyclic permutation of the blocks of a word in $\mathcal{W}_{n}$, can not be a non-primitive word.

\section{Proof of Theorem 1.1}

Let $\bar{B}(w)=\left(B_{i}(w)\right)_{i>0}$ be the sequence of blocks of $w$, ended by an infinite sequence of empty words, and let $\bar{r}(w)=\left(r_{i}(w)\right)_{i \geq 0}$ be the corresponding sequence of ranks.

Lemma 4.1. The weight $p(),. \bar{B}, \bar{r}$ and $N_{n}$ are $\mathfrak{C}_{n}$-measurable, and

$$
\mathbb{W}_{n}=\sum_{C \in \mathcal{C}_{n}} \frac{\operatorname{Card}(C) p(C)}{\mathbb{P}_{n}\left(\mathcal{W}_{n}\right)} \mathbb{U}_{C}
$$

Given that $w \in C$, the positions of the ranks of $w,\left(r_{i}(w)\right)_{1 \leq i \leq N_{n}(C)}$, are distributed uniformly on $\left\{1,2, \ldots, N_{n}(C)\right\}$.

Proof. The weight $p(w)$ depends only on the number of letters $a_{1}, a_{2}, \ldots$ that $w$ contains, not on the order of the letters in $w$, so that $p($.$) is constant on each$ $C \in \mathcal{C}_{n}$ : thus, under $\mathbb{W}_{n}$, the conditional distribution of $w$ given that $w \in C$ is $\mathbb{U}_{C}$. As a consequence of Proposition 3.2, the relation in Lemma 4.1 is just the disintegration of $\mathbb{W}_{n}$ according to its conditional distributions given $\mathfrak{C}_{n}$. Finally, $\mathbb{U}_{C(w)}$ is the image of the uniform probability on $\left[\mathfrak{S}_{N_{n}(w)} \times\{w\}\right] \cap \mathcal{W}_{n}$. Thus, by Propositions 3.1, 3.3 and Corollary 3.1, under $\mathbb{U}_{C(w)}$, the positions of the ranks are distributed uniformly on $\left\{1,2, \ldots, N_{n}(C)\right\}$. It follows that, under $\mathbb{W}_{n}$, the conditional distribution of the positions of the ranks given $C(w)$, or given $N_{n}$, is uniform too. 
We can see, by Lemma2.2 the probability that the number of blocks of a random word of $\mathcal{W}_{n}, N_{n}$, be of order $n$, increases when $n$ increases. Now, let the position of the $i$-th rank, denoted by $I_{n}$, is distributed uniformly on $\left\{1,2, \ldots, N_{n}(C)\right\}$. We put $\mathcal{I}_{n} \equiv \frac{2}{p_{1}\left(1-p_{1}\right) n} I_{n}$, the normalized position of the $i$-th rank by $\frac{p_{1}\left(1-p_{1}\right)}{2} n$. We shall see that $\mathcal{I}_{n}$ is approximately uniform, for its distribution is close to the uniform distribution on $[0,1]$, that we note $\mathbb{U}$ in the rest of paper. This proximity is understood with respect to the $\mathcal{L}_{2}$-Wasserstein metric $W_{2}\left(.\right.$, .). The $\mathcal{L}_{2}$-Wasserstein metric $W_{2}(.,$.$) is defined by$

$$
W_{2}(\mu, \nu)=\inf _{\substack{\mathcal{L}(X)=\mu \\ \mathcal{L}(Y)=\nu}} \mathbb{E}\left[\|X-Y\|_{2}^{2}\right]^{1 / 2},
$$

in which $\mu$ and $\nu$ are probability distributions on $\mathbb{R}^{d}$, and $\|\cdot\|_{2}$ denotes the Euclidean norm on $\mathbb{R}^{d}$. In this paper, we consider essentially the case $d=1$. We mention that convergence of $\mathcal{L}\left(X_{n}\right)$ to $\mathcal{L}(X)$ with respect to $W_{2}(.,$.$) entails convergence of X_{n}$ to $X$ in distribution, and we refer to 7 for an extensive treatment of Wasserstein metrics. In what follows, we shall improperly refer to the convergence of $X_{n}$ to $X$ with respect to $W_{2}(.,$.$) , meaning the convergence of their distributions. The main$ reason for the asymptotic uniformity of $\mathcal{I}_{n}$ is a form of convergence of the empirical distribution function $G_{n}(t)$ to $t$, in the notations of [9, Ch. 3.1, p.85, display (3)]:

Lemma 4.2. Consider a partition of $[0,1)$ into $n$ intervals $\left[\frac{i-1}{n}, \frac{i}{n}\right) ; i=1, \ldots, n$. For $\mathfrak{r}$ a random cyclic permutation of the class of all cyclic permutations of the intervals, we have

$$
W_{2}(\mathfrak{r}(i), \mathbb{U}) \leq \sqrt{1 / n} .
$$

This Lemma is also a specific case of [6, Lemma 6.3] where one can see for a proof.

Theorem 1.1 is demonstrated when the following proposition is proved:

Proposition 4.1. Let $\nu_{n}$ be the distribution of $\mathcal{I}_{n}$ under $\left(\mathcal{W}_{n}, \mathbb{W}_{n}\right)$. We have then:

$$
W_{2}\left(\nu_{n}, \mathbb{U}\right)=\mathcal{O}(\sqrt{1 / n}) .
$$

As a consequence, under $\mathbb{W}_{n}$, the moments of $\mathcal{I}_{n}$ converge to the corresponding moments of $\mathbb{U}$.

Proof. With the notations of Lemma 4.1, for $C \in \mathcal{C}_{n}$, let $\nu_{C}$ denote the image of $\mathbb{U}_{C}$ by $\mathcal{I}_{n}$, so that

$$
\nu_{n}=\sum_{C \in \mathcal{C}_{n}} \frac{\operatorname{Card}(C) p(C)}{\mathbb{P}_{n}\left(\mathcal{W}_{n}\right)} \nu_{C}
$$

Consider the blocks $b_{1} \leq \cdots \leq b_{N_{n}}$ of a word $w \in \mathcal{W}_{n}$, sorted in increasing lexicographic order. There exists at least one permutation $\tau \in \mathfrak{S}_{N_{n}}$ such that $w=b_{\tau(1)} \ldots b_{\tau\left(N_{n}\right)}$. Let $\mathfrak{R}_{N_{n}}$ denote the set of permutations $\delta$ such that $\omega=$ $\delta^{-1} \cdot \tau^{-1} . w$ be an element of $C(w)$. Then, for $\sigma$ is a random uniform element of $\mathfrak{R}_{N_{n}}$, $\omega=\sigma^{-1} \cdot \tau^{-1} \cdot w$ is a random uniform element of $C(w)$. Set $\Upsilon(\sigma)=\mathcal{I}_{n}\left(\sigma^{-1} \cdot \tau^{-1} \cdot w\right)$. Then, the distribution of $\Upsilon$ is $\nu_{C(w)}$ by Corollary 3.1 and Proposition 3.3. Thus, by a straightforward extension of Lemmas 4.2 and 2.2 ,

$$
W_{2}\left(\nu_{C(w)}, \mathbb{U}\right) \leq \mathbb{E}\left[(\Upsilon-U)^{2} \mid N_{n}^{a_{1}}>\frac{p_{1}\left(1-p_{1}\right)}{2} n\right]^{1 / 2} \leq \sqrt{\frac{2}{p_{1}\left(1-p_{1}\right) n}} .
$$


Finally, joining (3) and (4) we obtain

$$
W_{2}\left(\nu_{n}, \mathbb{U}\right)=\mathcal{O}(\sqrt{1 / n}) .
$$

and since $0 \leq \mathcal{I}_{n} \leq 1$, convergence of moments follows.

\section{REFERENCES}

[1] F. Bassino, J. Clément, and C. Nicaud, The standard factorization of Lyndon words: an average point of view, Discrete Math 290 (2005), 1-25.

[2] Ph. Chassaing, and E. Zohoorian-Azad, Asymptotic behavior of some factorizations of random words, submited to Ann. Appl. Probab. (online at arXiv, math.PR/1004.4062v1, 2010).

[3] M. Lothaire, Combinatorics on words, Encyclopedia of mathematics and its applications, 1983.

[4] M. Lothaire, Algebraic Combinatorics on Words, Cambridge University Press, V. 90 of Encyclopedia of mathematics and its applications, 2002.

[5] M. Lothaire, Applied Combinatorics on Words, Cambridge University Press, Encyclopedia of Mathematics and its Applications, 2005.

[6] R. Marchand, and E. Zohoorian-Azad, Limit law of the length of the standard right factor of a Lyndon word, Combinatorics Probability and Computing 16 (2007), 417-434.

[7] S.T. Rachev, Probability Metrics and the Stability of Stochastic Models, Wiley, Chichester, U.K., 1991.

[8] C. Reutenauer, Free lie algebras, Oxford Science Publications, London Mathematical Society Monographs New Series, 1993

[9] G.R. Shorack, J.A. Wellner, Empirical processes with applications to statistics, Wiley, 1986.

School of mathematics and COMputer sciences, Damghan university, Iran, P.o.Box 36716-41167

E-mail address: zohorian@du.ac.ir 